\title{
Los Determinantes de la Inversión Extranjera Directa en el Ecuador: Análisis Empírico del Periodo 2002-2017
}

The Determinants of Foreign Direct Investment in Ecuador: Empirical Analysis of the Period 2002-2017

Os determinantes do investimento estrangeiro direto no Equador: análise empírica do período 2002-2017

M.Sc. Mauricio Rivera P. mrivera@unach.edu.ec

Universidad Nacional de Chimborazo

ORCID: https://orcid.org/0000-0001-9612-7142

Lic. Daniel Solorzano Jiménez

ndsolorzano.fpe@unach.edu.ec

Universidad Nacional de Chimborazo

ORCID: https://orcid.org/0000-0003-0526-1712

M.Sc. Karol Pulgar S.

karitopulgar@gmail.es

Universidad Nacional de Chimborazo

ORCID: https://orcid.org/0000-0001-6260-9899

Christian Paúl Naranjo Navas, Ph.D.

cnaranjo@unach.edu.ec

Universidad Nacional de Chimborazo

ORCID: https://orcid.org/0000-0003-1532-203X

\section{RESUMEN}

El objetivo de esta investigación es identificar los determinantes que estimulan la inversión extranjera directa (IED) en Ecuador entre 2002 y 2017 . Mediante estimaciones de mínimos cuadrados ordinarios y un modelo de vectores autorregresivos (VAR) se comprueba empíricamente que el grado de apertura de la economía y la productividad del trabajo se relacionan positivamente con la IED, lo que indica que las inversiones de las empresas transnacionales (ET) están orientadas a la estrategia de búsqueda de mercados y eficiencia. Mientras tanto, fueron menos importantes el producto interno bruto, la inflación el tipo de cambio real y el costo laboral.

Palabras Clave: Inversión extranjera directa, crecimiento económico, determinantes, shocks.

\begin{abstract}
The objective of this research is to identify the determinants that stimulate foreign direct investment (FDI) in Ecuador between 2002 and 2017. Using ordinary least squares estimates and an autoregressive vector model (VAR), it is empirically verified that the degree of openness of the economy and labor productivity are positively related to FDI, which indicates that the investments of transnational companies (TNCs) are oriented towards the strategy of seeking markets and efficiency. Meanwhile, gross domestic product, inflation, the real exchange rate, and labor cost were less important
\end{abstract}

Keywords: Foreign direct investment, economic growth, determinants, shocks.

\section{RESUMO}

O objetivo desta pesquisa é identificar os determinantes que estimulam o investimento estrangeiro direto (IED) no Equador entre 2002 e 2017. Usando estimativas de mínimos quadrados ordinários e um modelo vetorial autorregressivo (VAR), verifica-se empiricamente que o grau de abertura do A economia e a produtividade do trabalho relacionam-se positivamente com o IDE, o que indica que os investimentos das empresas transnacionais (TNCs) estão orientados para a estratégia de procura de mercados e eficiência. Enquanto isso, o produto interno bruto, a inflação, a taxa de câmbio real e o custo da mão de obra eram menos importantes. 
Palavras-chave: Investimento estrangeiro direto, crescimento econômico, determinantes, choques.

\section{Introducción}

En un sistema monetario dolarizado como el ecuatoriano, en donde no se tiene injerencia en la emisión de dinero y por lo general las importaciones superan a las exportaciones, lo que provoca una salida permanente de divisas hacia el exterior, resulta de gran importancia desacelerar esta salida de recursos mediante la aplicación de diferentes mecanismos como el endeudamiento, las transferencias corrientes o la llegada de inversión extranjera directa (IED), que ocasiona la intención de atraer nuevos flujos de capitales, y de esa manera, asegurar una estabilidad en los principales indicadores macroeconómicos (VEINTIMILLA, 2015).

Muchos investigadores expresan que en la economía de los países desarrollados y sobre todo en aquellos en desarrollo como Ecuador, la IED se ha convertido en uno de los principales instrumentos para obtener recursos que promuevan el crecimiento económico. Mogrovejo (2005) expone que las naciones receptoras de IED, esperan obtener diferentes beneficios por este flujo de ingresos: reducción en las tasas de desempleo, acceso a nuevas tecnologías e ideas, mayor recaudación de impuestos y apertura de nuevas empresas industriales. Gil (2013) manifiesta que tener acceso a capital extranjero y sus inversiones, permiten a un país tener la oportunidad de desarrollar y fortalecer su economía; en el corto, mediano o largo plazo. Para Veintimilla (2015), mantener corrientes significativas de IED, se puede reflejar en un incremento de las exportaciones, esto como efecto de mayores niveles de productividad en las empresas, lo que permitirá un aumento del crecimiento económico y de la demanda agregada de un determinado país.

La IED puede generar efectos directos e indirectos en la economía de los países receptores de este tipo inversiones. En los efectos directos, se espera que la IED genere mayores niveles de empleo y producción, lo cual ocasionaría un impacto positivo y de incremento en el Producto Interno Bruto (PIB). Los efectos indirectos se encuentran determinados por la transferencia de tecnologías y nuevos conocimientos hacia las empresas locales (JIMÉNEZ y RENDÓN, 2012). Por lo tanto, el no contar con niveles altos y constantes de IED, podría dificultar a los diferentes países en el objetivo de fortalecer y desarrollar sus economías.

De acuerdo con el informe de la CEPAL (2018, p. 22) "en 2017 las entradas globales de IED disminuyeron (hasta 1,43 billones de dólares) por efecto de la caída del $37 \%$ en las economías desarrolladas, que ahora representan el 50\% de toda la IED recibida". Es así que en América Latina y el Caribe durante el mismo año, la IED también disminuyó en un 3.6\%, y con este resultado, fue el tercer periodo anual consecutivo en que los flujos de IED mantuvieron una tendencia decreciente en países de la región; esto ocasionado principalmente por el menor precio de los productos básicos de exportación y por la recesión económica en los años 2015 y 2016 (CEPAL, 2018).

En Ecuador, la IED en proporción al Producto Interno Bruto (PIB), ha registrado históricamente niveles muy reducidos, presentando un promedio anual del $1.12 \%$ durante el periodo comprendido entre los años 2002-2017. Veintimilla (2015) sostiene que los flujos recibidos de IED en Ecuador han sido generalmente bajos, en comparación con otros países de la región y en relación con el PIB local. Complementariamente, según datos del Banco Central, la IED atraída por Ecuador en el 2017 fue de 606 millones de dólares, lo cual es un equivalente al 0.6\% del tamaño de nuestra economía. Si se realiza esta misma relación en otros países de la región, observamos que nuestro país es uno de los que menos IED recibe en América Latina. A su vez, dentro del ámbito nacional, la IED captada en 2017 disminuyó un $20 \%$ en relación al periodo anterior. Esta disminución se originó fundamentalmente por problemas en el sector extractivo, "que en los dos años anteriores recibió alrededor de 500 millones de dólares anuales, pero al que solamente llegaron 64 millones de 
dólares en 2017" (CEPAL, 2018, p.52); siendo esta la principal causa en la caída significativa en la entrada de inversión extranjera directa a nuestro país.

En concordancia con los diferentes aportes que tiene la IED, la presente investigación procura identificar y explicar cuáles son los principales determinantes que motivan, estimulan o tienen en consideración los inversores extranjeros al momento de pensar invertir en nuestro país. De esta manera se podrá contar con evidencia empírica que permita realizar recomendaciones necesarias, para que Ecuador sea una potencial nación en la captación de inversión extranjera, lo cual permita un mayor nivel de desarrollo.

\section{Fundamentación Teórica}

Muchos estudios empíricos en diferentes países han tratado de encontrar los factores que influyen en la Inversión Extranjera. Correa, Días y Triches (2013) utilizaron un modelo econométrico de vectores de corrección de errores (VEC) para identificar los determinantes de la IED en Brasil, concluyendo que los niveles de la actividad económica se relacionan positivamente con la IED y que ésta misma relación existe con la productividad del trabajo y el salario. Por su parte, Gil, López y Espinosa (2013), mediante un modelo de panel de datos, establecieron que los países de América del Sur estudiados, que tuvieron una inflación controlada menor a un digito y un crecimiento constante del PIB, tuvieron una mayor recepción de IED. Romero y Estrada (2012), mediante un modelo de regresión lineal, observaron en el Ecuador el comportamiento de ciertas variables macroeconómicas, encontrando que la estabilidad de la inflación y el riesgo país, así como el incremento de las exportaciones son las que tienen mayor incidencia en el ingreso de inversión extranjera directa. Mottaleb y Kalirajan (2010) realizaron una investigaron en 68 países de Asia, África y América Latina utilizando datos de panel encontraron que la tasa de crecimiento del PIB, junto a un entorno favorable para las empresas y una moderna infraestructura (como teléfono e internet) son los factores más importantes para el ingreso de IED. La adopción de políticas comerciales orientadas hacia el exterior y un ambiente favorable para que los inversores extranjeros desarrollen sus actividades, pueden estimular sustancialmente la entrada de IED en los países en desarrollo.

\section{Inversión Extranjera Directa}

Existen diferentes teorías y estudios sobre los determinantes de la IED, Guerra (2001) sostiene que los primeros estudios se centraban en los incentivos y mecanismos que tenían las empresas para expandirse fuera de sus fronteras nacionales; posterior a esto, los estudios empezaron a examinar los factores o los motivos de localización de la IED. De acuerdo a la CEPAL (1997), existen tres teorías primarias sobre la movilización de la IED: internalización de las empresas transnacionales, paradigma ecléctico de la producción internacional y la teoría macroeconómica de la IED. La teoría de la Internalización de las Empresas Transnacionales parte de los postulados de Ronald Coase (1937), que según Veintimilla (2015), el autor determina que las empresas trasnacionales internacionalizaran su producción de bienes finales o intermedios para maximizar sus beneficios si, al evaluar los costos de transacción de desarrollar las actividades productivas, los que se presentan en un país extranjero son menores a los que se obtendrían en el país de origen.

Eden y Dai (2010), Palilla y Gomes (2015), Tomelin et al (2018), y UNCTAD (1998) explican que el Paradigma Ecléctico de la Producción Internacional (Modelo OLI) fue propuesto por John Dunning, según este modelo, si una empresa encuentra ventajas en el exterior en materia de propiedad (ownership) y ubicación (location), las empresas aprovecharan dichas ventajas si la producción se internaliza mediante la inversión extranjera directa (AGUILAR HUERTA, 2004). En cuanto a la Teoría Macroeconómica, el ideólogo de esta teoría es Kiyoshi Kojima, quien manifiesta 
que la inversión extranjera directa va al país receptor que tiene ventajas comparativas en relación al país inversor en ciertas industrias o sectores. Villareal (2004), complementa esta idea indicando que para el enfoque macroeconómico "los flujos de capital tendrían lugar cuando el capital desplazado pueda ser combinado con los factores de producción existentes en el país receptor de los flujos tal que se consignan unos menores costos de producción" (p. 252).

Los estudios en países en desarrollo y economías emergentes presentan los siguientes elementos para atraer la inversión extranjera: la estabilidad económica y política, una adecuada infraestructura, costos laborales bajos, entre otros. Existen estudios como el de Sekkat, Khalid y Veganzones-Varoudakis, Marie-Ange (2007), que determinan la importancia de la apertura comercial, la disponibilidad de la infraestructura y la buena situación económica y política como condiciones importantes para aumentar el atractivo de los países en desarrollo con respecto a la IED, especialmente en los países del sur de Asia, África y Oriente Medio y que existe un mayor impacto en la IED en el sector manufacturero que en la IED total. Vijayakumar, Sridharan, y Chandra Sekhara Rao (2010) elaboran un estudio para los BRICS utilizando análisis de datos del Panel para el período 1975 a 2007 y encuentran que las variables tamaño del mercado, costo laboral, infraestructura, valor de la moneda y formación de capital bruto son los determinantes más importantes para la entrada de recursos. Por otro lado, Gupta y Singh (2016), estudian el período 1983-2013, con un modelo de efectos aleatorios modificados, el cual revela que el índice de producción industrial (IPI), tasas de inflación, tasas de desempleo, apertura comercial, el tipo de cambio efectivo real y costo laboral son los determinantes para atraer entradas de IED en los países BRICS.

También se han realizado estudios empíricos que buscan determinar los factores que influyen en el ingreso de flujos externos, utilizando efectos fijos estándar y un panel dinámico, realizado por Economou, Hassapis, Philippas, y Tsionas (2016) para 24 países de la OCDE y 22 países en desarrollo (no miembros de la OCDE) durante 1980-2012, determinan que el tamaño del mercado, la formación bruta de capital y los impuestos corporativos son determinantes fuertes para el ingreso de IED en los países de la OCDE, mientras que en los países en desarrollo, son importantes el tamaño del mercado, el costo laboral y las variables institucionales. Además, Agiomirgianakis et. al. (2003) concluyen que, en un estudio realizado para 20 países de la OCDE, entre 1975 y 1997, ciertas variables, como el capital humano, el régimen comercial y la densidad de la infraestructura fueron significativos para explicar el ingreso de la IED; también se observa la importancia positiva del factor de aglomeración. Finalmente, Alam et. al. (2013) identificaron que, en un estudio para el período 1985-2009, para un panel de diez países de la OCDE, el tamaño del mercado, el costo de la mano de obra y la calidad de la infraestructura son determinantes en la IED, adicionalmente se establece una relación bidireccional a corto plazo entre el tamaño del mercado y los costos laborales, mientras que la calidad de la infraestructura influye en el tamaño del mercado y los costos laborales a corto plazo.

Otros estudios importantes son los elaborados por Gómez Aguirre, Windler Muñoz y Massa Roldán (2020), que explican que otros factores que impulsan el ingreso de IED se encuentra a apertura comercial, con énfasis en las importaciones. Por su parte, Sánchez Navarro (2013), establece que el tamaño de mercado de la economía receptora (reflejado en el PIB), el mercado potencial (medido por al tamaño de las economías cercanas) y el riesgo de la inversión en economías circundante, son grandes determinante en las dinámicas de atracción de IED. En un estudio para México, Elizalde, Arana y Martínez (2020), concluyen que los factores importantes de ingreso de IED están relacionados con el criterio de eficiencia (con base en las variables precio del petróleo, nivel de endeudamiento), que resultó relevante comparado con los criterios de disponibilidad de recursos, dimensión del mercado y factor riesgo económico. Un trabajo elaborado para El Salvador por Sierra (2020), concluye que los determinantes que incentivan al ingreso de capitales a países en desarrollo son: el tamaño del mercado, el costo de la mano de obra, un buen ambiente institucional económico-político y, la calidad de la infraestructura. Finalmente, Ávila-López, Galván-León y Zayas-Márquez (2020), elaboran un estudio para encontrar los 
factores que influyen el ingreso de IED China en Latinoamérica, concluyendo que la única variable que por el momento atrae la inversión de este país es el tamaño de la economía, es decir, aquellos países que cuenten con un PIB más elevado son más atractivos para el ingreso de capitales.

\section{Metodología}

Para establecer la relación existente entre la IED y sus determinantes se utilizó un modelo de vectores autorregresivos (VAR); la data corresponde a series trimestrales, en número índice, desde el año 2002 a 2017, obtenidas de fuentes como el Banco Central del Ecuador (BCE) y el Instituto Nacional de Estadística y Censos (INEC), que se las transformó a logaritmos.

Según Arias y Torres (2004), en un VAR "todas las variables son consideradas como endógenas, pues cada una de ellas se expresa como una función lineal de sus propios valores rezagados y de los valores rezagos de las restantes variables del modelo" (p. 3). La representación matemática de un VAR es la siguiente:

$$
Y_{t}=A_{1} Y_{t-1}+\cdots+A_{p} Y_{t-p}+B X_{t}+\epsilon_{t}
$$

Donde:

$Y_{t}=$ Es un vector $k$ de variables endógenas.

$X_{t}=$ Vector de variables exógenas.

$A_{1}, \ldots, A_{p}$ y $B=$ Matrices de coeficientes

$\epsilon_{t}=$ Vector de errores o innovaciones que pueden estar contemporáneamente correlacionados; pero no auto correlacionados con sus propios rezagos.

El procedimiento que se desarrolló para la estimación econométrica inicio al obtener el Test de Levene, para conocer si es necesario realizar una transformación logarítmica en las diferentes series. A continuación, mediante una regresión de mínimos cuadros ordinarios (MCO), se verificó si las variables seleccionadas son significativas como determinantes de la inversión extranjera directa en Ecuador. Posteriormente se aplicó el test de raíz unitaria, a través del estadístico Dickey Fuller Aumentado (ADF), para conocer si las series son, o no estacionarias. Con base en los análisis anteriores se procede a desarrollar el modelo (VAR), esto en cuanto en niveles hubo variables que presentaron estacionaridad, lo que es un indicio fehaciente para utilizar el modelo ya mencionado.

En primera instancia es necesario determinar el número óptimo de retardos a fin de identificar cuantos valores rezagados se deben introducir en el modelo. Posteriormente se realizó la prueba de causalidad de Granger, la cual busca determinar estadísticamente si los valores pasados y presentes de una variable $X$ contienen información que contribuya a explicar el comportamiento de la variable $Y$. En otras palabras, permite identificar como una variable $X$ causa o predice a otra variable $Y$; a su vez, este mismo escenario pude darse de forma inversa. En la etapa final del modelo VAR se analizó la función impulso respuesta (FIR) y la descomposición de la varianza, que según Loría (2007), la FIR es la respuesta de la variable dependiente ante choques en los términos de error o innovaciones en las variables endógenas y la descomposición de la varianza es un análisis complementario a la FIR y permite medir el porcentaje de volatilidad que registra una variable por cambios o choques en las demás.

Para determinar la relación de cointegración de las variables, se desarrolló un modelo de vectores de corrección de errores (VEC), que es una adaptación para el Ecuador de la especificación empírica propuesta por Correa, Dias y Triches (2013), para identificar y evaluar los determinantes del flujo de inversión extranjera directa hacia el Brasil entre 2001 y 2013. 
El modelo VAR queda definido de la siguiente manera:

$$
I E D_{t}=\beta_{0}+\beta_{1} P I B_{t}+\beta_{2} I N F_{t}+\beta_{3} G A E_{t}+\beta_{4} T C R_{t}+\beta_{5} C T_{t}+\beta_{1} P T_{6 t}+u_{t}
$$

Donde:

$\mathrm{IED}=$ Inversión Extranjera Directa Bruta, variable endógena.

$\mathrm{PIB}=$ Producto Interno Bruto.

$\mathrm{INF}=$ Tasa de Inflación, Índice de Precios al Consumidor.

GAE = Grado de Apertura de la Economía: calculado por la razón entre la suma de las exportaciones e importaciones en términos FAO y el valor del PIB en términos corrientes.

TCR= Tipo de Cambio Real.

$\mathrm{CL}=$ Costo Laboral: medido mediante el salario real. empleados.

PT = Productividad del Trabajo: calculado entre la razón del PIB real y el número de

$\mathrm{u}_{\mathrm{t}}=\quad$ Término de perturbación estocástico

Por la naturaleza de la investigación, en la que se utilizan datos de series temporales no es necesario el test de heterocedasticidad, por cuanto este test suele ser frecuente en datos de panel y de corte transversal. Adicionalmente, las variables tuvieron transformación logarítmica, lo que ayuda a estabilizar la varianza.

\section{Resultados y discusión}

La Inversión Extranjera Directa IED (IED) ha registrado fluctuaciones volátiles entre 2002 y 2017. En los tres primeros años existieron ingresos altos y estables por un valor de 2.492 millones de dólares, debido a que se inició la construcción del Oleoducto de Crudos Pesados (OCP) y se desarrollaron proyectos de exploración y explotación de campos petrolífero por parte de empresas transnacionales. Entre 2005 y 2006 la IED disminuyó como consecuencia de la declaración de nulidad del contrato con la empresa petrolera Occidental por haber violado los términos estipulados de exploración y explotación. En 2008 la IED fue de 1.057 millones de dólares la segunda cifra más alta entre 2002 y 2017, debido al "incremento de la inversión en los sectores de transporte, almacenamiento y comunicaciones, impulsado por las inversiones de América Móvil y Telefónica" (CEPAL, 2008, p. 27).

En 2009, la IED disminuye en un 70\%, profundizando más este decrecimiento con la IED de 166 millones de dólares recibida en 2010, que fue el valor más bajo en el periodo, debido al rediseño en los contratos con las grandes compañías petroleras, el cual estipulaba que el gobierno es el propietario del petróleo bombeado y pagaría a las empresas una tarifa por barril extraído, lo que ocasionó la salida de varias transnacionales del país (CEPAL, 2010). Durante el 2011-2017 el ingreso de IED se vio influenciado por la construcción de grandes proyectos en el sector público y por el programa de políticas que buscaban modificar la matriz productiva del país, en donde uno de los anuncios del gobierno fue la puesta en marcha del "Proyecto de Promoción y Atracción de Inversiones" que tuvo como objetivo "incrementar de manera significativa los flujos de IED que recibe el país en un conjunto de sectores priorizados: turismo, metalmecánica, energía y servicios, entre otros" (CEPAL,2012, p.32).

En 2015 la IED Ecuador registró una cifra record en las entradas de IED, con un monto de 1.322 millones de dólares fue la más alta durante el periodo de estudio. Este aumento según la (CEPAL, 2016) fue producto de importantes fusiones y adquisiciones transfronterizas como la 
compra del 64\% de la Unión Cementera Nacional (UCEM) por un valor 230 millones de dólares; y la concesión del Aeropuerto Mariscal Sucre de Quito por un monto de 232 millones de dólares. Por último, en 2017 la IED recibida disminuyó un 20\% en relación al periodo anterior. Esta disminución se originó fundamentalmente por problemas en el sector extractivo, "que en los dos años anteriores recibió alrededor de 500 millones de dólares anuales, pero al que solamente llegaron 64 millones de dólares en 2017" (CEPAL, 2018, p.52).

En el periodo 2002-2017 el total de la IED recibida en Ecuador fue de 10.402 millones de dólares, mostrándose la mayor concentración en la explotación de minas y canteras con un $40 \%$ del total de la IED recibida, seguida de por la industria manufacturera con un $18 \%$ y el sector del comercio, que representa un $15 \%$ de participación. Este total recibido de IED proviene de 52 países: 22 de América, 17 de Europa, 9 de Asia, 2 de África y 2 de Oceanía. En este contexto la inversión proveniente de 12 países, representó el 84\% del total de la IED recibida entre 2002 y 2017. Dentro de los principales países inversores tenemos a México con un 14\%, de los cuales el $96 \%$ de esta inversión fue en el sector de las telecomunicaciones. Brasil con el 10\%, 95\% de sus recursos destinados a la actividad minera y petrolera. Panamá con el $9 \%$, de estos un $37 \%$ se invierten en industria manufacturera, $23 \%$ en comercio, $18 \%$ en servicios de empresas y $17 \%$ en el sector agrícola del país. Países Bajos, Canadá, China y España con un promedio del $8.4 \%$ referente a la IED global, realizaron inversiones en el sector de las minas y canteras primordialmente y en servicios prestados a las empresas.

\section{Producto Interno Bruto Real}

El Producto Interno Bruto entre 2002 y 2017 tuvo un incremento promedio de $3.8 \%$ en el periodo analizado. En 2004, el PIB registró su mayor tasa de crecimiento, con un $8.2 \%$ debido al aumento del valor agregado petrolero. Durante los años 2005, 2006 y 2007 los incrementos de la economía ecuatoriana son leves, volviendo a tener un crecimiento significativo de 6.4\%. en el 2008. Sin embargo, y como consecuencia de la grave crisis mundial generada por el colapso de la burbuja inmobiliaria en Estados Unidos, provocó que para el 2009 apenas exista el 0.6\% de incremento. A partir de esto la economía creció considerablemente los dos años siguientes como resultado del aumento en los precios del petróleo y un fuerte gasto del gobierno, especialmente en infraestructura pública. No obstante, desde el año 2012 las tasas de crecimiento de la economía empiezan a disminuir entre cada periodo, esto producto de la caída en los precios del petróleo, devolución de la moneda en países fronterizos, catástrofe natural (terremoto), entre otros factores, desencadenaron que la economía ecuatoriana sufra un decrecimiento del -1.2\% en el 2016.

\section{Inflación}

La inflación durante el periodo analizado entre 2002 y 2017 tuvo un valor promedio del $4.39 \%$. En el año 2000, Ecuador adoptó la dolarización y, como efecto, la inflación entre 2000 y 2004 fue alta, con cifras del $95.51 \%, 40.26 \%, 12.55 \%$ y $7.95 \%$ respectivamente. A partir de este año, la inflación anual bajó drásticamente y mantuvo incrementos menores al 5\%, a excepción del año 2008 que presentó una inflación del $8.39 \%$ debido a la crisis internacional, que se originó en Estados Unidos y luego se esparció al resto del mundo. Por el contrario, el menor nivel de inflación se registra en 2017, (0.42\%), que según Alberto Acosta-Burneo citado en France24 (2018) esta variación se debe a la gran cantidad de petrodólares que ingresaron en la economía, lo que originó una proliferación de créditos lo cual impulsó el consumo, provocando que el país se encarezca. 


\section{Índice del Tipo de Cambio Real}

Entre el 2000 y el 2003 el Tipo de cambio real (TCR), se encontraba en un proceso de estabilización producto de la dolarización a inicios del milenio. En 2009 se detecta una apreciación que para Araque, Rivera, y Argüello (2017) fue consecuencia de los shocks en los términos de intercambio, producto del aumento de los precios de los commodities. Hasta el 2011 existe un alza del TCR, resultado de shocks positivos en los precios del petróleo y un fuerte incremento en el gasto del gobierno. Durante el 2012 y 2016 se origina una caída continua del TCR, básicamente por la valoración del dólar a nivel internacional, caída del precio del petróleo y devaluación de la moneda en varios países con los que Ecuador comercializa.

\section{Grado de Apertura de la Economía}

El grado de apertura de la economía ecuatoriana (GAE) durante el periodo de estudio registró un valor promedio de 47\%: Entre 2002-2008 mantuvo una tendencia creciente debido a que en esta etapa las exportaciones consiguieron la mayor tasa de crecimiento entre 2002 y 2017 , siendo el año 2008 el de mayor apertura, pero que disminuyó en el año 2009, al igual que otros indicadores macroeconómicos debido a la crisis financiera mundial. Sin embargo, en 2016 y como consecuencia de la apreciación del dólar y la caída en los precios del petróleo el GAE presentó el menor registro con un porcentaje del 32\%. En 2017 el GAE de Ecuador fue de 37\% .

\section{Costo Laboral}

En promedio durante el 2002-2017 el salario nominal tuvo una tasa de crecimiento del 9.5\%, mientras que la del salario real fue de $17.13 \%$. Para estos salarios los años de mayor crecimiento fueron el 2002 y 2008, ambos como consecuencia de la alta inflación que se registró en estos años. A su vez entre el 2002 y 2017 la diferencia promedio entre el salario nominal con respecto al real, fue de $\$ 40.43$, lo que significa que; al recibir el salario monetario, una persona perdió su poder adquisitivo en $\$ 40$.

\section{Productividad del Trabajo}

La productividad del trabajo creció en un promedio anual del 1.3\% entre el 2002 y el 2017. Los mayores aumentos se registraron en el 2004, 2008 y 2011, consecuencia del incremento en el valor agregado petrolero y un fuerte gasto del gobierno, especialmente en infraestructura pública. En 2009 tuvo hubo un decrecimiento del $-1.4 \%$, como consecuencia de la grave crisis mundial, generada por el colapso de la burbuja inmobiliaria en Estados Unidos. A partir del 2012 las tasas de crecimiento fueron disminuyendo entre cada periodo, llegando al -3\% en 2016 y al $-5.5 \%$ en 2017 , esto producto de la caída en los precios del petróleo, apreciación del dólar a nivel internacional, catástrofe natural (terremoto), entre otros factores.

\section{Estimación Econométrica}

$$
I E D_{t}=\beta_{0}+\beta_{1} P I B_{t}+\beta_{2} I N F_{t}+\beta_{3} G A E_{t}+\beta_{4} T C R_{t}+\beta_{5} C L_{t}+\beta_{1} P T_{6 t}+u_{t}
$$

\section{Test de Levene}

Se establecen las siguientes hipótesis: 
$H_{0}:$ Varianzas constantes

$H_{1}:$ Varianzas no constantes

\begin{tabular}{lc}
\multicolumn{2}{c}{ Tabla.1. Test de Levene } \\
\hline & Probabilidad \\
\hline IED & 0.0204 \\
PIB & 0.7339 \\
INF & 0.0537 \\
GAE & 0.0175 \\
TCR & 0.0001 \\
CL & 0.0015 \\
PT & 0.0138 \\
\hline
\end{tabular}

Las probabilidades de las variables IED, GAE, TCR, CL y PT son menores al valor de significancia del 5\% y se rechaza la hipótesis nula, que implica que las series no presentan varianzas constantes y es necesario aplicar una transformación logarítmica en ellas. El PIB e INF muestran evidencia en favor de la $H_{0}$, por tener un valor mayor al 5\%, pero también serán transformadas en logaritmo, puesto que basta con que una de las variables incluidas en el modelo sea menor al de significancia, para que se proceda a realizar la transformación en todas las demás.

Con la transformación logarítmica, el modelo econométrico es el siguiente:

$$
\begin{aligned}
& \text { LOGIED }_{t=} \beta_{0}+\beta_{1} \text { LOGPIB }_{t}+\beta_{2} \text { LOGINF }_{t}+\beta_{3} L_{\text {LOGGAE }}+\beta_{4} \text { LOGTCR }_{t}+\beta_{5} \text { LOGCL }_{t}+ \\
& \beta_{1} \text { LOGPT }_{6 t}+u_{t}
\end{aligned}
$$

\section{Ecuación mínimos cuadrados ordinarios}

Las variables exógenas son significativas al 5\%, es decir que aportan al comportamiento de la inversión extranjera directa. Además de aquello se aprecia que las variables LOGGAE y LOGPT presentan una relación directa con la variable endógena, mientras que LOGPIB, LOGINF, LOGTCR y LOGCL mantienen una relación inversa con la IED.

Tabla 2. Regresión mediante Mínimos Cuadrados Ordinarios

\begin{tabular}{lcc}
\hline Variable & Coeficiente & Prob. \\
\hline LOGPIB & -22.29488 & 0.0039 \\
LOGINF & -1.445523 & 0.0395 \\
LOGGAE & 2.628454 & 0.0208 \\
LOGTCR & -6.371472 & 0.0305 \\
LOGCL & -0.808262 & 0.0343 \\
LOGPT & 13.4355 & 0.0117 \\
C & 75.03711 & 0.0353 \\
\hline R-cuadrado & 0.220416 & \\
R-ajustado & 0.136889 & \\
F-estadística & 2.638868 & \\
Prob (F-estadística) & 0.025226 & \\
Estadística de Durbin- & 2.553861 & \\
Watson & & \\
\hline
\end{tabular}

El valor de $R^{2}=0.22$ es menor que el estadístico Durbin-Watson $=2.55$ y se descarta la presencia de una regresión espuria; por lo tanto, la relación de las variables es lógica y verdadera. 


\section{Test de Raíz Unitaria}

Con el estadístico Dickey Fuller Aumentado (ADF), se determina las propiedades de estacionaridad, aplicando el test de raíz unitaria. Para aquello se forman las siguientes hipótesis:

\section{$H_{0}$ : Existe Raiz Unitaria-La Serie no es Estacionaria \\ $H_{1}$ : No Existe Raiz Unitaria- La Series es Estacionaria}

La condición para aceptar o rechazar la hipótesis nula $\left(H_{0}\right)$ es la siguiente:

Si, $\begin{aligned} A D F_{\text {Calculado }} & >\text { Valor Crítico } \rightarrow \text { No Rechazo } H_{0} \\ \text { Si } \quad A D F_{\text {Prob }} & <\text { Valor Crítico } \rightarrow \text { Rechazo } H_{0}\end{aligned}$

Previo a establecer los resultados del Dickey Fuller Aumentado, se utiliza el estadístico de Durbin-Watson (DW), para determinar autocorrelación. La tabla 4, muestra que las variables LOGPIB, LOGGAE, LOGTCR, LOGCL y LOGPT no presentan problemas de autocorrelación; sin embargo, LOGIED y LOGINF presentaron dicho problema, por lo que fue necesario introducir especificaciones a estas dos últimas variables, y de esa manera lograr que se encuentren dentro del intervalo recomendado de 1.85 y 2.15 .

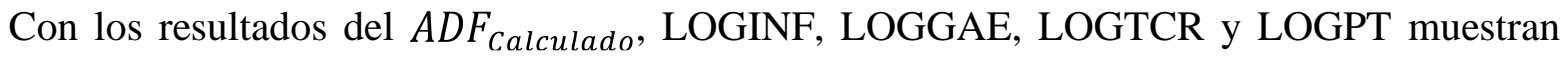
que son series no estacionarias en niveles; por el contrario, los resultados de LOGIED, LOGPIB y LOGCL determinan que son series estacionarias en sus niveles.

Tabla 1. Test de Raíz Unitaria-ADF-en Niveles

\begin{tabular}{lcccccc}
\hline \multirow{2}{*}{ Variable } & $\mathrm{ADF}_{\text {Calculado }}$ & $\begin{array}{c}\text { Valor } \\
\text { Crítico 5\% }\end{array}$ & DW & Retardos & $\mathrm{ADF}_{\text {Prob }}$ & Diagnostico \\
& & & & & & \\
\hline LOGIED & -4.496125 & -2.911730 & 1.889644 & 3 & 0.0006 & $\mathrm{I}(0)$ \\
LOGPIB & -5.242935 & -2.909206 & 2.045261 & 0 & 0.0000 & $\mathrm{I}(0)$ \\
LOGINF & -1.853372 & -2.910019 & 2.015195 & 1 & 0.3518 & $\mathrm{I}(1)$ \\
LOGGAE & -1.495709 & -2.913549 & 1.993557 & 5 & 0.5286 & $\mathrm{I}(1)$ \\
LOGTCR & -1.362034 & -2.910019 & 1.869965 & 1 & 0.5950 & $\mathrm{I}(1)$ \\
LOGCL & -6.897544 & -2.911730 & 1.932884 & 3 & 0.0000 & $\mathrm{I}(0)$ \\
LOGPT & -2.478803 & -2.911730 & 1.877363 & 3 & 0.1257 & $\mathrm{I}(1)$ \\
\hline
\end{tabular}

El cuanto a las series que no fueron estacionarias en sus niveles se procedió a estimar el test de raíz unitaria con una primera diferencia. No se encontraron problemas de autocorrelación y las series LOGINF, LOGGAE, LOGTCR y LOGPT muestran que son estacionarias en primeras diferencias y por lo tanto poseen un nivel de integración de orden uno I(1).

Anexo 1. Test de Raíz Unitaria-ADF-en Primeras Diferencias

\begin{tabular}{lcccccc}
\hline Variable & $\mathrm{ADF}_{\text {Calculado }}$ & $\begin{array}{c}\text { Valor } \\
\text { Crítico 5\% }\end{array}$ & DW & Retardos & ADF $_{\text {Prob }}$ & Diagnostico \\
\hline LOGINF & -3.996340 & -2.910860 & 1.910945 & 2 & 0.0027 & $\mathrm{I}(0)$ \\
LOGGAE & -3.834189 & -2.913549 & 2.040078 & 4 & 0.0045 & $\mathrm{I}(0)$ \\
LOGTCR & -4.791538 & -2.910860 & 2.016295 & 2 & 0.0002 & $\mathrm{I}(0)$ \\
LOGPT & -9.359565 & -2.911730 & 1.960107 & 2 & 0.0000 & $\mathrm{I}(0)$ \\
\hline
\end{tabular}

\section{Modelo de Vectores Autorregressivos (VAR)}

Debido a que unas series si presentan y otras no presentan estacionariedad, no existe evidencia de una relación de equilibrio en el largo plazo (cointegración). A continuación, se 
determinó que el número óptimo de retardos es cinco y el modelo VAR considera que la cantidad de información que se queda en los residuos es mínima.

Tabla 2. Determinación del Número Óptimo Rezagos

\begin{tabular}{ccccccc}
\hline Lag & LogL & LR & FPE & AIC & SC & HQ \\
\hline 0 & 524.7863 & NA & $4.15 \mathrm{E}-17$ & -17.8547 & -17.60603 & -17.75784 \\
1 & 839.2602 & 542.1964 & $4.45 \mathrm{E}-21$ & -27.00897 & -25.01958 & -26.23406 \\
2 & 976.1695 & 203.0035 & $2.31 \mathrm{E}-22$ & -30.04033 & $-26.31022^{*}$ & -28.58737 \\
3 & 1072.698 & 119.828 & $5.44 \mathrm{E}-23$ & -31.67923 & -26.2084 & -29.54823 \\
4 & 1137.47 & 64.7727 & $4.77 \mathrm{E}-23$ & -32.22312 & -25.01157 & -29.41407 \\
5 & 1255.659 & $\mathbf{8 9 . 6 6 0 0 0} *$ & $\mathbf{9 . 6 2 e - 2 4} *$ & $\mathbf{- 3 4 . 6 0 8 9 2} *$ & -25.65665 & $\mathbf{- 3 1 . 1 2 1 8 3} *$ \\
\hline
\end{tabular}

\section{Prueba de Causalidad de Granger}

El test de causalidad de Granger presenta las siguientes hipótesis:

$H_{0}$ : No existe causalidad en el sentido de Granger

$H_{1}$ : Existe causalidad en el sentido de Granger

La condición para aceptar o rechazar la hipótesis nula $\left(H_{0}\right)$ es la siguiente:

$$
\begin{aligned}
\text { Si, } \quad \text { Prob }>0.05 & \rightarrow \text { No Rechazo } H_{0} \\
\text { Si, } \quad \text { Prob } & <0.05 \rightarrow \text { Rechazo } H_{0}
\end{aligned}
$$

Con los resultados obtenidos existe evidencia que el PIB, el tipo de cambio real, el costo del trabajo y la productividad del trabajo no causan en el sentido de Granger a la inversión extranjera directa. Complementariamente, la inflación y el grado de apertura de la economía causan en el sentido de Granger a la IED. Es importante señalar que existe una causalidad unidireccional entre todas las variables mencionadas.

Tabla 3. Prueba de Causalidad de Granger Dependent variable: LOGIED

\begin{tabular}{lccc}
\hline \multicolumn{1}{c}{ Excluded } & Chi-sq & df & Prob. \\
\hline LOGPIB & 3.443566 & 5 & 0.6319 \\
LOGINF & 15.95631 & 5 & 0.0070 \\
LOGGAE & 14.43865 & 5 & 0.0131 \\
LOGTCR & 7.836052 & 5 & 0.1655 \\
LOGCL & 7.714798 & 5 & 0.1727 \\
LOGPT & 11.02004 & 5 & 0.0510 \\
\hline All & 61.96332 & 30 & 0.0005 \\
\hline
\end{tabular}

\section{Función Impulso-Respuesta}

La respuesta de la inversión extranjera directa (LOGIED) ante choques o innovaciones de sus determinantes en un periodo de 10 trimestres (dos años y medio). Se observa que ante un shock en el producto interno bruto la IED responde de manera positiva los tres primeros periodos, a partir de ahí decrece en el cuarto y vuelve a crecer en el quinto, posterior aquello presenta tendencias decrecientes y crecientes a lo largo de los periodos. En cuanto a un choque en la inflación, la IED responde de forma negativa en los dos primeros trimestres, para luego crecer en el tercer periodo a partir del cual pasa a mantener una tendencia decreciente el resto del tiempo. La respuesta de la IED ante un cambio en el grado de apertura de la economía es negativa durante el primer semestre, luego pasa a crecer hasta el quinto trimestre, y después de esto empieza a fluctuar de manera positiva y negativa el resto de periodos.

Por su parte, un shock en el tipo de cambio real provoca que la IED sea negativa durante los dos primeros periodos, para luego aumentar y disminuir el resto de los trimestres. Un cambio en el 
costo laboral conduce a que la IED sea decreciente en el primer semestre, luego pasa a crecer en el tercer periodo, donde a partir de esto, empieza a decrecer y crecer el resto del tiempo. Por último, la respuesta de la IED frente a un choque en la productividad del trabajo es positiva durante los dos primeros periodos, después disminuye y aumenta hasta el séptimo trimestre para pasar a tener una tendencia constante los tres últimos periodos.

\section{Descomposición de la Varianza}

La descomposición de la varianza permite identificar que, en el corto plazo, la IED varia en $5.62 \%$ por choques en el producto interno bruto, el $7.13 \%$ por cambios en la inflación, el 3.14\% por shocks en el grado de apertura de la economía, el $10.24 \%$ por innovaciones en el tipo de cambio real, el $3.90 \%$ por choques en el costo laboral y el $1.86 \%$ por shocks en la productividad del trabajo. Mientras que en el largo plazo y siguiendo la misma secuencia anterior, la IED varia en el $8.42 \%$, el $8.49 \%$, el $9.05 \%$, el $12.22 \%$, el $3.99 \%$ y el $2.80 \%$ por choques en cada uno de sus determinantes.

Tabla 4. Descomposición de la Varianza

\begin{tabular}{cccccccc}
\hline Period & LOGIED & LOGPIB & LOGINF & LOGGA & LOGTC & LOGCL & LOGPT \\
\hline 1 & 100.0000 & 0.000000 & 0.000000 & 0.000000 & 0.000000 & 0.000000 & 0.000000 \\
4 & 68.09189 & 5.622645 & 7.132320 & 3.145332 & 10.24079 & 3.905887 & 1.861143 \\
10 & 55.00574 & 8.421362 & 8.498917 & 9.052392 & 12.22908 & 3.991489 & 2.801019 \\
\hline
\end{tabular}

\section{Conclusiones}

En la estimación econométrica se empleó un modelo de vectores autorregresivos (VAR), se comprueba empíricamente que el grado de apertura de la economía y la productividad del trabajo se relacionan positivamente con el ingreso de IED; mientras tanto, aunque menos relevante el producto interno, la inflación, el tipo del cambio real y el costo laboral también fueron estadísticamente significativos para explicar el comportamiento de los niveles de IED recibida en Ecuador. Debido a que en principio las variables no fueron significativas, se las debió transformar a números índices, para que el modelo sea adecuado.

La evolución de los niveles de IED tuvo fluctuaciones volátiles entre 2002 y 2017, que se vieron influenciadas primordialmente por la inversión en actividades: petroleras y mineras, industria manufacturera y de comercio. En al año 2010 se registró el nivel de ingreso más bajo de IED consecuencia del rediseño en el contrato con las compañías petroleras, lo que ocasionó la salida de varias transnacionales del país. Todo lo contrario, sucedió en 2015, que producto de importantes fusiones y adquisiciones transfronterizas, la IED alcanzó una cifra record de 1.322 millones de dólares, siendo este el valor más alto durante el periodo de investigación.

El comportamiento de los determinantes de la IED (Producto Interno Bruto, Inflación, Tipo de Cambio Real, Grado de Apertura de la Economía, Costo Laboral y Productividad del Trabajo) en su gran mayoría se vieron afectados por acontecimientos similares: los efectos que sufrió la economía ecuatoriana a inicios del nuevo milenio como consecuencia de la dolarización; la crisis internacional, en 2008, que se originó en Estados Unidos y se expandió al resto del mundo; y por último, entre 2012 y 2017 los determinantes de la IED se vieron influenciados por: shocks en los términos de intercambio, caída en los precios del petróleo, apreciación del dólar a nivel mundial; y el terremoto que soportó Ecuador en abril de 2016.

Finalmente, este estudio debido a su naturaleza, no contempla entornos que son muy importantes en el ingreso de flujos de recursos a países como el Ecuador; entre estos ámbitos se encuentran determinantes administrativos (por ejemplo, desarrollo de la industria bancaria, apertura comercial), jurídicos (como costos por incumplimiento de contratos, costo de registrar una propiedad y otros), o de influencia del estado de la economía de países vecinos (relación espacial), 
que al conjuntarlos en un modelo econométrico podrían entregar resultados más completos. Estos nuevos elementos y factores que influyen en el ingreso de IED, establecen la posibilidad de un mayor abanico de posibilidades de nuevos estudios, especialmente para nuestro país.

\section{REFERENCIAS}

AGIOMIRGIANAKIS, George; ASTERIOU, Dimitrios y PAPATHOMA, K. The Determinants of Foreign Direct Investment: A Panel Data Study for the OECD Countries. Londrés, UK.: Department of Economics, City University London, 2003.

AGUILAR HUERTA, Isaías. Planteamientos básicos del enfoque OLI y nuevas estrategias de las corporaciones transnacionales. Aportes: Revista de la Facultad de Economía-BUAP., VIII (24), 77-91, 2004.

ALAM, Abdullah y ZULFIQAR ALI SHAH, Syed. Determinants of foreign direct investment in OECD member countries. Journal of Economic Studies, 40 (4), 515-527, 2013.

AL-SHIBAMI, Ahmed Hamoud. Location related determinants of Foreign Direct Investment in Yemen: Dunning's Eclectic Paradigm perspective. Australian Journal of Basic and Applied Sciences, 5(8), 394-404, 2011.

ARAQUE, Wilson; RIVERA, Jairo y ARGÜELLO, Andrés. Tipos de Cambio Real de Equilibrio para Ecuador: Período 2000-2015. Cuestiones Económicas, 27(1:2), 47-74, 2017.

ARIAS, Eilyn y TORRES, Carlos. Modelos VAR y VECM para el pronóstico de corto plazo de las importaciones de Costa Rica. Documento de Trabajo del Banco Central de Costa Rica, (22), 2004.

Ávila-López, Luis, Galván-León, Jorge y ZayasMárquez, Carolina. (2020). Determinantes de la inversión extranjera directa China en Latinoamérica. Quipukamayoc, 25(58), 43-49.

BANCO CENTRAL DEL ECUADOR. Notas metodológicas de la balanza de pagos del Ecuador, 2018. Obtenido de https://contenido.bce.fin.ec/documentos/Estadi sticas/SectorExterno/BalanzaPagos/metodolog ia.pdf

BANCO CENTRAL DEL ECUADOR. Inversión Extranjera Directa: Por Modalidad de Inversión, Rama de Actividad Económica y País de Origen, 2019. Obtenido de https://contenido.bce.fin.ec/documentos/Estadi sticas/SectorExterno/BalanzaPagos/InversionE xtranjera/Directa/indice.htm

BANCO CENTRAL DEL ECUADOR. Preguntas Frecuentes Banco Central del Ecuador, 2019. Obtenido de https://www.bce.fin.ec/index.php/component/ $\mathrm{k} 2 /$ item/156-preguntas-frecuentes-bancocentral-del-ecuador
BRUGGER, Samuel. Capital Especulativo y Crisis Bursátil en América Latina. Contagio, Crecimiento y Convergencia (tesis doctoral). Universidad Autónoma de México, 2010.

CALLEN, Tim. What is gross domestic product? Finance \& Development, 45(4), 48-49, 2008.

CASTRO, Pricila; FERNANDES, Elain y CAMPOS, Antonio. Os determinantes do investimento direto estrangeiro no Brasil e no México: uma análise empírica. Associação Nacional dos Centros de Pós-Graduação em Economia, 2013. Obtenido de https://anpec.org.br/encontro/2013/files_I/i72bf472e0d97d8e932bc37c86feac6d31.pdf

COASE, Ronald The Nature of the Firm. Económica, 4 (16), 386-405, 1937.

\section{COMISIÓN ECONÓMICA PARA AMÉRICA} LATINA Y EL CARIBE (CEPAL). La Inversión Extranjera Directa en América Latina y el Caribe 2010 . Santiago de Chile : LC/G.2494-P, 2010.

COMISIÓN ECONÓMICA PARA AMÉRICA LATINA Y EL CARIBE (CEPAL). La Inversión Extranjera Directa en América Latina y el Caribe 2015. Santiago de Chile : LC/G.2680-P, 2016

COMISIÓN ECONÓMICA PARA AMÉRICA LATINA Y EL CARIBE (CEPAL). La Inversión Extranjera en América Latina y el Caribe 1997. Santiago de Chile: LC/G.1985-P, 1998.

COMISIÓN ECONÓMICA PARA AMÉRICA LATINA Y EL CARIBE (CEPAL). La Inversión Extranjera Directa en América Latina y el Caribe 2008. Santiago de Chile: LC/G.2406-P, 2008.

COMISIÓN ECONÓMICA PARA AMÉRICA LATINA Y EL CARIBE (CEPAL). La Inversión Extranjera Directa en América Latina y el Caribe 2012. Santiago de Chile: LC/G.2571-P, 2012.

\section{COMISIÓN ECONÓMICA PARA AMÉRICA} LATINA Y EL CARIBE (CEPAL). La Inversión Extranjera Directa en América Latina y el Caribe 2017. Santiago de Chile: LC/PUB.2018/13-P, 2018.

CONNEY, Marulanda. Análisis de series temporales con R (II): Estacionariedad y raíces unitarias, (11 de marzo de 2018. Obtenido de FinanzasZone: http://finanzaszone.com/analisis-y-prediccion- 
de-series-temporales-con-r-ii-estacionariedady-raices-unitarias/

CORRERA, Eduarda Martins, DIAS, Jorge y TRICHES, Divanildo. Los determinantes de la inversión extranjera directa en el Brasil: análisis empírico del período 2001-2013. Revista de la CEPAL, (121), 186-189, 2017.

DE LA GARZA, Ulises. La importancia de la inversión extranjera directa en México. InnOvaciOnes de NegOciO, 3(1), 55 - 68, 2006.

DUNNING, John y LUNDAN. Institutions and the OLI paradigm of the multinational enterprise. Asia Pacific Journal of Management, 25(4), 573-593, 2008

DUNNING, John. Trade, Location of Economic Activity and the MNE: A Search for an Eclectic Approach. In: Ohlin, B., Hesselborn, P.O. and Wijkman, P.M., Eds.,. The International Allocation of Economic Activity, 395-418, 1977.

DUNNING, John. The eclectic paradigm of international production: a restatement and some posible extensions. Journal of International Businees Studies, 19(1), 1-31, 1998.

DUNNING, John. The Eclectic (OLI) Paradigm of International Production: Past, Present and Future. International Journal of the Economics of Business, 8 (2), 173-190, 2001.

ECONOMOU, Fotini; HASSAPIS, Cristis; PHILIPPAS, Nicholaos y TSIONAS. Foreign Direct Investment Determinants in OECD and Developing Countries. Review of Development Economics, 21(3), 2016.

EDEN, Lorraine y DAI, Li. Rethinking the $\mathrm{O}$ in Dunning's OLI/Eclectic Paradigm. Multionational Business Review, 18(1), 1334, 2010.

Elizalde, Hector, Arana, José y Martínez, Miguel. (2020). Determinantes macroeconómicos de la inversión extranjera directa en México 20002016. Análisis Económico, XXXV(89), 117 142.

FLORA, Preeti y AGRAWAL, Gaurav. Determinants of Direct foreign Investment as a Means of International Market Entry: A Review. Indian Journal of Economics and Development, 2(4), 63-83, 2014.

FRANCE 24. Inflación de Ecuador de -0,20\% en 2017 la más baja en una década, 6 de enero de 2018 . Obtenido de https://www.france24.com/es/20180106inflacion-de-ecuador-de-020-en-2017-la-masbaja-en-una-decada

GIL, Edgar; LÓPEZ, Silvio y ESPINOSA, Dorian. Factores determinantes de la Inversión Extranjera Directa en América del Sur. Perfil de Coyuntura Económica, (22), 55-85, 2013.
GÓMEZ AGUIRRE, Regina, WINDLER MUÑOZ, Lia y MASSA ROLDÁN, Ricardo. (2020). Determinantes de la inversión extranjera directa en América Latina: una visión desde la economía administrativa. Revista Economía y Política. (31).

GUERRA, Alfredo. Factores determinantes de la inversión extranjera: introducción a una teoría inexistente. Revista del Banco Nacional de Comercio Exterior: México, 2001. Obtenido de

http://revistas.bancomext.gob.mx/rce/magazin es/32/8/guer0901.pdf

GUPTA, Priya y SINGH, Archana. Determinants of Foreign Direct Investment Inflows in BRICS Nations: A Panel Data Analysis. Emerging Economy Studies, 2(2), 181-198, 2016.

INSTITUTO NACIONAL DE ESTADÍSTICA Y CENSOS. Índice de Precios al Consumidor, 2019. Obtenido de http://www.ecuadorencifras.gob.ec/indice-deprecios-al-consumidor/

JIMÉNEZ, Dora y RENDÓN, Hernando. Determinantes y efectos de la Inversión Extranjera Directa: revisión de literatura. Ensayos de Economía, 22(41), 109-128, 2012.

KRUGMAN, Paul y OBSTFELD, Maurice. Economía Internacional. Teoría y política. (7ma ed.). Madrid, España: Pearson Educación S.A., 2006.

LEVY, Eduardo, STEIN, Ernesto y DAUDE, Chistian. Regional Integration and the Location of FDI. Working Paper, Inter-American Development Bank, Research Department, 2003.

LORÍA, Eduardo. Econometría con Aplicaciones. México: Pearson Educación, 2007.

MAGALHÃES, Karla. La mundialización de la economía brasileña en sus empresas transnacionales (tesis doctoral). Universidad de Barcelona: España, 2015.

MOGROVEJO, Jesús. Factores Determinantes de la Inversión Extranjera Directa en algunos países de Latinoamérica. Estudios Económicos de Desarrollo Internacional., 5-2, 63-94, 2005.

MORALES, Josefina. Inversión extranjera directa y desarrollo en América Latina. Problemas del Desarrollo. Revista Latinoamericana de Economía, 41(163), 141-156, 2010.

MOTTALEB, Khondoker y KALIRAJAN, Kaliappa. Determinants of Foreign Direct Investment in Developing Countries: A Comparative Analysis. Margin the Journal of Applied Economic Research, 4(4), 369-404, 2010.

NAYAK, Dinkar y CHOUDHURY, Rahul. A selective review of foreign direct investment theories. Economic and Social Commission for Asia and the Pacific, 2014. Obtenido de https://www.unescap.org/sites/default/files/A WP\%20No.\%20143_0.pdf 
ORGANISATION FOR ECONOMIC COOPERATION AND DEVELOPMENT (OECD). Foreign Direct Investment in Developing Countries: Determinants and Impact, 2001. Obtenido de https://www.oecd.org/daf/inv/investmentstatist icsandanalysis/2407305.pdf

ORGANISATION FOR ECONOMIC COOPERATION AND DEVELOPMENT (OECD) Inversiones extranjeras directas en desarrollo: un máximo de beneficios por un costo mínimo. Resumen. París: OECD, 2002.

PADILLA, Ramón y GOMES, Caroline.

Determinantes de la salida de IED y efectos en el país emisor. CEPAL Serie Estudios y Perspectivas, $\mathrm{N}^{\circ}$ 166. México, 2015.

PARKIN, Michael. Macroeconomía. Séptima edición. México: Pearson Educación, 2007.

PAZ, Jorge. Apertura y crecimiento: lo que muestran los datos, 2019. Obtenido de El Economista: https://www.eleconomista.com.ar/2019-06apertura-y-crecimiento-lo-que-muestran-losdatos/

RAMÍREZ, Carlos y FLOREZ, Laura. Aplicaciones de inversión extranjera directa: definiciones, tipoligía y casos de aplicación colombianos, 2006. Obtenido de Departamento de Economía del Instituto Colombiano de Estudios Superiores de Incolda: https://repository.icesi.edu.co/biblioteca_digit al/bitstream/10906/2363/1/ramirez_apuntes_in version_2006.pdf

RIVAS ACEVES, Salvador y PUEBLA MÉNEZ, Alondra. Inversión Extranjera Directa y Crecimiento Económico. Revista Mexicana de Economía y Finanzas, 11(2), 51-75, 2016.

RODRÍGUEZ, Fernando. Metodología de cálculo de los índices de tipo de cambio real del Ecuador, s.f. Obtenido de Banco Central del Ecuador: https://contenido.bce.fin.ec/documentos/Publi cacionesNotas/Catalogo/Cuadernos/Cuad119. pdf

ROMERO, Jorge y JARAMILLO, Edison. Factores Determinantes de la Inversión Extranjera Directa en el Ecuador Periodo 1992-2010 (tesis de pregrado). Universidad del Azuay, Ecuador, 2012.

SÁNCHEZ, Luis. Estudio Económico de la Industria Farmacéutica y su Proceso de Patentamiento: Efecto en el nivel de Producción (2007-2013) (tesis de posgrado). Instituto Politécnico Nacional: México, D.F., 2015.
SÁNCHEZ, Dennis. Determinantes de los flujos de inversión extranjera estadounidense a través de un modelo gravitacional con componente espacial: evidencia para algunos países latinoamericanos. Ensayos sobre Política Económica, 31(72), 35-50, 2013.

SARAVIA, Alejandra. "Determinantes de la Inversión Extranjera Directa (IED) en Bolivia 19962008", 2009. Obtenido de Banco Central de Bolivia: https://www.bcb.gob.bo/eeb/sites/default/files/ archivos2/D2M1P3\%20Saravia.pdf

SEKKAT, Khalid y VEGANZONES-VAROUDAKIS, Marie-Ange. Openness, Investment Climate, and FDI in Developing Countries. Review of Development Economics, 11(4), 607-620, 2007.

SIERRA, Carlos. (2020). Caracterización y determinantes de la inversión extranjera directa en El Salvador. San Salvador: REDIBACEN- Banco Central de la República del El Salvador.

SISTEMA DE INDICADORES SOCIALES DE ECUADOR. Salario Real, 2019. Obtenido de http://www.siise.gob.ec/siiseweb/PageWebs/S ISSAN/ficsan_K033.htm

TOMELIN, Jurema; AMAL, Mohamed; HEIN, Nelson y CARPES. Foreign direct investment in the G-20: to what extent do institutions matter? RAUSP Management Journal, 53(3), 404-421, 2018.

UNITED NATIONS CONFERENCE ON TRADE AND DEVELOPMENT. World Investment Report 1998: Trend and Determinants. New York and Geneva, 1998.

VEINTIMILLA, Juan. Determinantes de la Inversión Extranjera Directa en Ecuador: Aplicación de un Modelo de Gravedad. Periodo 2002 - 2014 (tesis de posgrado). Facultad Latinoamericana de Ciencias Sociales Sede Ecuador, 2015.

VIJAYAKUMAR, Narayanamurthy; SRIDHARAN, Perumal y CHANDRA SEKHARA RAO, Kode. Determinants of FDI in BRICS Countries: A panel analysis. International Journal of Business Science and Applied Management, 5(3), 1-13, 2010.

VILLAREAL, C. Las teorías de la localización de la inversión extranjera directa (IED): una aproximación. Innovaciones de Negocios, 1(2), 245-263, 2004. 